\title{
Runge-Kutta integrators yield optimal regularization schemes
}

\author{
Andreas Rieder \\ Institut für Praktische Mathematik und Institut für Wissenschaftliches Rechnen und \\ Mathematische Modellbildung, Universität Karlsruhe, 76128 Karlsruhe, Germany \\ E-mail: andreas.rieder@math.uni-karlsruhe.de
}

Received 11 October 2004, in final form 8 December 2004

Published 21 January 2005

Online at stacks.iop.org/IP/21/453

\begin{abstract}
Asymptotic regularization (also called Showalter's method) is a theoretically appealing regularization scheme for an ill-posed problem $T x=y, T$ acting between Hilbert spaces. Here, $T x=y$ is stably solved by evaluating the solution of the evolution equation $u^{\prime}(t)=T^{*}(y-T u(t)), u(0)=0$, at a properly chosen finite time. For a numerical realization, however, we have to apply an integrator to the ODE. Fortunately all properties of asymptotic regularization carry over to its numerical realization: Runge-Kutta integrators yield optimal regularization schemes when stopped by the discrepancy principle. In this way a common analysis is obtained for such different regularization schemes as, for instance, the Landweber iteration and the iterated Tikhonov-Phillips method which are generated by the explicit and implicit Euler integrators, respectively. Furthermore it turns out that inconsistent Runge-Kutta schemes, which are useless for solving ODEs, lead to optimal regularizations as well which can even be more efficient than regularizations from consistent Runge-Kutta integrators. The presented computational examples illustrate the theoretical findings and demonstrate that implicit schemes outperform the explicit ones.
\end{abstract}

\section{Introduction}

We are interested in the stable solution of the linear system

$$
T f=g^{\varepsilon}
$$

where the operator $T$ acts continuously between the Hilbert spaces $X$ and $Y$. The right-hand side $g^{\varepsilon}$ is a perturbed version of the exact data $g$ satisfying $\left\|g^{\varepsilon}-g\right\|_{Y} \leqslant \varepsilon$ where $\varepsilon \geqslant 0$ is the noise level. We will assume that $g=T f^{+}$with $f^{+}$in $\mathrm{N}(T)^{\perp}$, the orthogonal complement of the null space of $T$. Moreover, the above linear system is considered to be ill posed, that is, the range $\mathrm{R}(T)$ of $T$ is non-closed in $Y$. 
Special procedures have to be used for obtaining stable and meaningful approximations to $f^{+}$. Those procedures are called regularization schemes (regularizations). Let $\left\{\mathcal{R}_{n}\right\}_{n \in \mathbb{N}_{0}}$ be a family of continuous operators (linear or nonlinear) from $Y$ to $X$ with $\mathcal{R}_{n} 0=0$. If there exists a mapping $\gamma:] 0, \infty\left[\times Y \rightarrow \mathbb{N}_{0}\right.$ such that the worst reconstruction error tends to zero, i.e.

$$
\sup \left\{\left\|f^{+}-\mathcal{R}_{\gamma\left(\varepsilon, g^{\varepsilon}\right)} g^{\varepsilon}\right\|_{X}: g^{\varepsilon} \in Y,\left\|T f^{+}-g^{\varepsilon}\right\|_{Y} \leqslant \varepsilon\right\} \longrightarrow 0 \quad \text { as } \quad \varepsilon \rightarrow 0
$$

for any $f^{+} \in \mathrm{N}(T)^{\perp}$, then the pair $\left(\left\{\mathcal{R}_{n}\right\}_{n \in \mathbb{N}_{0}}, \gamma\right)$ is a regularization scheme for $T$. The mapping $\gamma$ is called parameter choice.

The above convergence is arbitrarily slow unless $f^{+}$satisfies additional requirements. Convergence rates can be given, for instance, when $f^{+}$is in the source set $X_{\mu, \varrho}:=\left\{|T|^{\mu} w\right.$ : $\left.w \in \mathrm{N}(T)^{\perp},\|w\|_{X} \leqslant \varrho\right\}$ for $\mu, \varrho>0$ where $|T|:=\left(T^{*} T\right)^{1 / 2}$. We distinguish regularization schemes attaining the highest convergence speed in $\mathrm{X}_{\mu, \varrho}$ by the concept of order optimality: the regularization scheme $\left(\left\{\mathcal{R}_{n}\right\}_{n \in \mathbb{N}_{0}}, \gamma\right)$ for $T$ is called of optimal order in $\mathrm{X}_{\mu, \varrho}$ if $^{1}$

$\sup \left\{\left\|f^{+}-\mathcal{R}_{\gamma\left(\varepsilon, g^{\varepsilon}\right)} g^{\varepsilon}\right\|_{X}: f^{+} \in \mathrm{X}_{\mu, \varrho}, g^{\varepsilon} \in Y,\left\|T f^{+}-g^{\varepsilon}\right\|_{Y} \leqslant \varepsilon\right\} \leqslant C_{\mu} \varepsilon^{\mu /(\mu+1)} \varrho^{1 /(\mu+1)}$.

The constant $C_{\mu}$ depends neither on $\varepsilon$ nor on $\varrho$.

A common way to obtain and to analyse potential regularization schemes is using filter functions: let $\left\{F_{n}\right\}_{n \in \mathbb{N}_{0}}$ be a family of piecewise continuous functions $F_{n}:\left[0,\|T\|^{2}\right] \rightarrow \mathbb{R}$ with jump discontinuities satisfying

$\lim _{n \rightarrow \infty} F_{n}(\lambda)=1 / \lambda \quad$ and $\quad \lambda\left|F_{n}(\lambda)\right| \leqslant C_{F} \quad$ for $\left.\left.\quad \lambda \in\right] 0,\|T\|^{2}\right]$.

Defining $\mathcal{R}_{n}:=F_{n}\left(T^{*} T\right) T^{*} \in \mathcal{L}(Y, X)$ we have a candidate for a regularization scheme which needs to be furnished with a parameter choice. As parameter choice $\gamma$ we will use, exclusively throughout the paper, the easy-to-implement discrepancy principle of Morozov: choose a $\tau>1$ and set

$$
\gamma\left(\varepsilon, g^{\varepsilon}\right):=\min \left\{n \in \mathbb{N}_{0}:\left\|T \mathcal{R}_{n} g^{\varepsilon}-g^{\varepsilon}\right\|_{Y} \leqslant \tau \varepsilon\right\} .
$$

The pair $\left(\left\{\mathcal{R}_{n}\right\}_{n \in \mathbb{N}_{0}}, \gamma\right)$ is a regularization scheme for $T$ indeed (see, e.g., $\left.[2,11]\right)$.

The order optimality of $\left(\left\{\mathcal{R}_{n}\right\}_{n \in \mathbb{N}_{0}}, \gamma\right)$ can be read from the qualification of the underlying filter functions which we introduce now: due to the convergence in (1.2) the supremum of the set $\left\{\left|F_{n}(\lambda)\right|: 0 \leqslant \lambda \leqslant\|T\|^{2}\right\}$ grows unboundedly with $n$. Thus, there exists a sequence $\left\{t_{n}\right\}_{n \in \mathbb{N}_{0}}$ which diverges to infinity monotonically such that $\sup \left\{\left|F_{n}(\lambda)\right|: 0 \leqslant \lambda \leqslant\|T\|^{2}\right\}=$ $\mathrm{O}\left(t_{n}\right)$ as $n \rightarrow \infty$. The qualification $\mu_{\mathrm{Q}}$ of the filter $\left\{F_{n}\right\}_{n \in \mathbb{N}_{0}}$ is the largest number such that, for all $\left.\mu \in] 0, \mu_{\mathrm{Q}}\right]$,

$$
\sup _{0 \leqslant \lambda \leqslant\|T\|^{2}} \lambda^{\mu / 2}\left|1-\lambda F_{n}(\lambda)\right| \leqslant C_{Q} t_{n}^{-\mu / 2} \quad \text { as } n \rightarrow \infty .
$$

Here, $C_{Q}$ may depend on $\mu$.

Filters furnished with the discrepancy principle yield regularizations of optimal order. A proof of the following theorem can be found in [2, theorem 4.17] or in [11, Satz 3.4.1].

Theorem 1.1. Let $T \in \mathcal{L}(X, Y)$ and let $\left\{F_{n}\right\}_{n \in \mathbb{N}_{0}}$ be a filter with qualification $\mu_{\mathrm{Q}}>1$. The corresponding sequence $\left\{t_{n}\right\}_{n \in \mathbb{N}_{0}}$ is assumed to fulfil $t_{n} / t_{n+1} \geqslant \vartheta>0$. Let the parameter choice $\gamma$ be the discrepancy principle with $\left.\tau>\sup \left\{\left|1-\lambda F_{n}(\lambda)\right|: n \in \mathbb{N}_{0}, 0 \leqslant \lambda \leqslant\|T\|^{2}\right]\right\} \geqslant 1$.

Then, $\left(\left\{\mathcal{R}_{n}\right\}_{n \in \mathbb{N}_{0}}, \gamma\right), \mathcal{R}_{n}:=F_{n}\left(T^{*} T\right) T^{*}$, is a regularization scheme for $T$ of optimal order in $\mathrm{X}_{\mu, \varrho}$ for all $\left.\left.\mu \in\right] 0, \mu_{\mathrm{Q}}-1\right]$ and all $\varrho>0$.

Let us look at three examples of regularization schemes. All details and more examples can be found, e.g., in $[2,11]$.

1 The given exponents of $\varepsilon$ and $\varrho$ are indeed the largest possible, see, e.g., [2, section 3.2] or [11, Kap. 3.2.3]. 
Example 1.2. Let $\left\{t_{n}\right\}_{n \in \mathbb{N}_{0}}$ be a sequence monotonically diverging to infinity and satisfying the requirements of theorem 1.1 .

1. The filter $F_{n}(\lambda)=1 /\left(\lambda+t_{n}^{-1}\right)$ leads to the Tikhonov-Phillips regularization where $\mathcal{R}_{n}=F_{n}\left(T^{*} T\right) T^{*}=\left(T^{*} T+t_{n}^{-1} I\right)^{-1} T^{*}$. The qualification of the method is $\mu_{\mathrm{Q}}=2$.

2. Consider the evolution equation

$$
u^{\prime}(t)=T^{*}(y-T u(t)), \quad u(0)=0,
$$

which has a unique solution $u:\left[0, \infty\left[\rightarrow X\right.\right.$. Setting $\mathcal{R}_{n} y:=u\left(t_{n}\right)$ we obtain Showalter's method, also known as asymptotic regularization. This method has an infinite qualification, that is, $\mu_{\mathrm{Q}}=\infty$ as can be seen from $\mathcal{R}_{n}=F_{n}\left(T^{*} T\right) T^{*}$ with the filter

$$
F_{n}(\lambda)= \begin{cases}\left(1-\mathrm{e}^{-\lambda t_{n}}\right) / \lambda & : \quad \lambda>0, \\ t_{n} & : \quad \lambda=0 .\end{cases}
$$

3. The $v$-methods $(v>0)$ are semi-iterative schemes. For scaled $T$, that is, $\|T\| \leqslant 1$, the $n$th iterate $f_{n}$ can be represented by $f_{n}=\mathcal{R}_{n} y=F_{n}\left(T^{*} T\right) T^{*} y$ with $F_{n}(\lambda)=\left(1-\widetilde{P}_{n}^{v}(\lambda)\right) / t$ where $\widetilde{P}_{n}^{v}(\lambda)=P_{n}^{(2 v-1 / 2,-1 / 2)}(1-2 \lambda) / P_{n}^{(2 v-1 / 2,-1 / 2)}(1)$ and $P_{n}^{(a, b)}$ denotes the $n$ th-order Jacobi polynomial.

The $v$-method has finite qualification $\mu_{\mathrm{Q}}=2 v\left(t_{n}=n^{2}\right)$.

In the remainder of this paper we will show that Runge-Kutta integrators, applied to the initial value problem (1.5) and stopped by the discrepancy principle, are regularization schemes for $T$ being of optimal order in $\mathrm{X}_{\mu, \varrho}$ for all $\mu, \varrho>0$, that is, $\mu_{Q}=\infty$.

Remark 1.3. Already at this early stage we take the opportunity to emphasize an important difference in using Runge-Kutta integrators for solving the ODE (1.5) and for regularizing the ill-posed problem (1.1). While in the former case we like to approximate the solution $u$ accurately over a time interval, we are, in the latter situation, interested in finding quickly a stable approximation of the stationary solution of (1.5). In our subsequent investigations it will therefore turn out that low order, even inconsistent, Runge-Kutta schemes in combination with large time steps are best suited for our purpose.

The paper is organized as follows. The next section introduces Runge-Kutta integrators together with those of their properties which we will need later on. Our above-announced main result is stated and validated in section 3. Moreover, we give examples of Runge-Kutta integrators some of which lead to well-known iterative regularization schemes. In section 4 we discuss implementation issues and study the performance of different Runge-Kutta schemes for solving numerically an integral equation of the first kind. A close look at the proof of our main result allows for a generalization covering so-called inconsistent Runge-Kutta schemes (section 5). Thus, we can design regularization methods converging faster (in a specific sense) than regularizations from consistent Runge-Kutta integrators. In the final section we comment on exponential integrators and nonlinear problems.

\section{Runge-Kutta integrators}

In this section we recall those well-known properties of Runge-Kutta methods which will be of interest later in the paper. For details and proofs, see, e.g., [1, 3, 9].

Runge-Kutta methods belong to the class of one step methods for the numerical solution of initial value problems for ODEs,

$$
w^{\prime}(t)=\Psi(t, w(t)), \quad t>0, \quad w(0)=w_{0},
$$

with given $\Psi:\left[0, \infty\left[\times W \rightarrow W\right.\right.$ and $w_{0} \in W$. Here, $W$ is a Banach space. 
A Runge-Kutta method of $s$ stages with variable time steps $\left.\left\{\Delta \mathrm{t}_{n}\right\}_{n \in \mathbb{N}} \subset\right] 0, \infty[$ provides approximations $w_{n}$ to $w\left(t_{n}\right), t_{n}=\sum_{k=1}^{n} \Delta \mathrm{t}_{k}$, by

$$
\begin{aligned}
& w_{n}=w_{n-1}+\Delta \mathrm{t}_{n} \sum_{i=1}^{s} b_{i} k_{i}\left(t_{n-1}, w_{n-1}, \Delta \mathrm{t}_{n}\right), \\
& k_{i}=\Psi\left(t_{n-1}+c_{i} \Delta \mathrm{t}_{n}, w_{n-1}+\Delta \mathrm{t}_{n} \sum_{j=1}^{s} a_{i j} k_{j}\right), \quad i=1, \ldots, s .
\end{aligned}
$$

The given coefficients $A=\left\{a_{i j}\right\} \in \mathbb{R}^{s \times s}, b=\left(b_{1}, \ldots, b_{s}\right)^{t}$ and $c=\left(c_{1}, \ldots, c_{s}\right)^{t}$ determine the particular method. The method is called explicit if $A$ is a strictly lower triangular matrix. Otherwise, the method is called implicit, since linear or nonlinear algebraic equations have to be solved to retrieve the stages $k_{i}, i=1, \ldots, s$.

Runge-Kutta methods are compactly represented by the Butcher array

$$
\begin{array}{c|c|ccc}
c & A \\
\hline & b^{t}
\end{array}=\begin{array}{cccc}
c_{1} & a_{11} & \cdots & a_{1 s} \\
\vdots & \vdots & \vdots & \vdots \\
c_{s} & a_{s 1} & \cdots & a_{s s} \\
\hline & b_{1} & \cdots & b_{s}
\end{array} .
$$

Application of a Runge-Kutta integrator to a linear autonomous ODE, that is, $\Psi(t, w(t))=$ $M w(t)+v$ in (2.1), yields the recursion

$$
w_{n}=R\left(\Delta \mathrm{t}_{n} M\right) w_{n-1}+\Delta \mathrm{t}_{n} Q\left(\Delta \mathrm{t}_{n} M\right) v
$$

where

$$
\begin{aligned}
& R(z)=1+z b^{t}(I-z A)^{-1} \mathbb{1}, \\
& Q(z)=b^{t}(I-z A)^{-1} \mathbb{1}=(R(z)-1) / z, \\
& \mathbb{1}=(1, \ldots, 1)^{t} \in \mathbb{R}^{s} .
\end{aligned}
$$

The function $R$ is called the stability function of the method. It is a polynomial for explicit methods and it is a rational function for implicit methods. The Runge-Kutta method is said to be of order $p$ if the following asymptotic relation holds true:

$$
R(z)=\mathrm{e}^{z}+\mathrm{O}\left(z^{p+1}\right) \quad \text { as } \quad z \rightarrow 0 .
$$

If the stability region

$$
S=\{z \in \mathbb{C}:|R(z)| \leqslant 1\}
$$

contains the left complex half-plane $\mathbb{C}_{-}$, the Runge-Kutta scheme is said to be A-stable. An A-stable method with $|R(\infty)|<1$ is called strongly A-stable. If we even have $|R(\infty)|=0$, an A-stable method is L-stable. Strong A-stability and L-stability are crucial properties for integrating stiff ODEs coming from parabolic problems. Our equation (1.5) can be considered parabolic with $T^{*} T$ as elliptic component.

Example 2.1. Here we list some well-known Runge-Kutta schemes with their stability functions and their respective orders. For details see, e.g., [1, 3, 9].

1. The implicit Euler scheme is represented by

$$
\begin{array}{l|l}
1 & 1 \\
\hline & 1
\end{array}, \quad R(z)=\frac{1}{1-z}, \quad p=1 .
$$

Its stability region is $S=\mathbb{C}_{-}$and we have $|R(\infty)|=0$. Thus, it is L-stable. 
2. The SDIRK2 method ${ }^{2}$ with two stages is given by

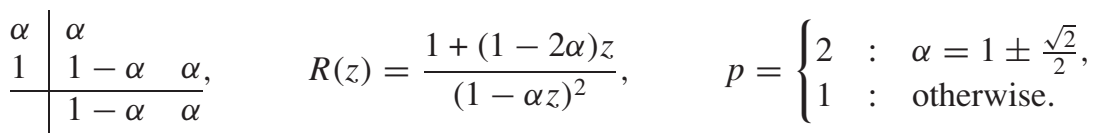

It is L-stable for $1-\sqrt{2} / 2 \leqslant \alpha \leqslant 1+\sqrt{2} / 2$.

3. The explicit Euler scheme is represented by

$$
\begin{array}{l|l}
0 & 0 \\
\hline & 1
\end{array}, \quad R(z)=1+z, \quad p=1 .
$$

Its stability region is the disc $S=\{z \in \mathbb{C}:|z+1| \leqslant 1\}$ with radius 1 centred about -1 .

4. Since the stability region of an explicit method is always compact one can ask for its real stability boundary $\beta_{R}<\infty$. By definition, $\left[-\beta_{R}, 0\right]$ is the largest segment of the negative real axis contained in $S$. For any explicit Runge-Kutta scheme of order one at least we have that $\beta_{R} \leqslant 2 s^{2}$ where $s$ is the stage number. The optimal real stability boundary, i.e., $\beta_{R}=2 s^{2}$, is attained for the optimal polynomials $R(z)=\mathrm{T}_{s}\left(1+z / s^{2}\right)$ where $\mathrm{T}_{s}$ is the first kind Chebyshev polynomial of order $s$, see, e.g., [9, theorem V.1.1]. Please note that $\mathrm{T}_{1}(1+z)=1+z$ is the stability function of the explicit Euler scheme.

For the optimal polynomials of order $s \geqslant 2$ there exist inner points $z \in]-2 s^{2}, 0[$ where $|R(z)|=1$. A little damping of the optimal polynomials shrinks the maximal stability boundary a little bit, but yields on the other hand that $|R(z)|<1$ for all $z \in]-\beta_{R}, 0\left[\right.$ : choose $\omega_{0}>1$, but close to 1 , and set

$$
R(z)=\mathrm{T}_{s}\left(\omega_{0}+\omega_{1} z\right) / \mathrm{T}_{s}\left(\omega_{0}\right), \quad \omega_{1}:=\mathrm{T}_{s}\left(\omega_{0}\right) / \mathrm{T}_{s}^{\prime}\left(\omega_{0}\right) .
$$

Here, $\beta_{R}=2 \omega_{0} / \omega_{1}$. For instance, choosing $\omega_{0}=1+\delta / s^{2}$ for a positive but small $\delta$, we obtain $\beta_{R} \approx(2-4 \delta / 3) s^{2}$.

There are several ways to construct Runge-Kutta schemes possessing the abovedamped polynomials as stability functions. The approach of van der Houwen and Sommeijer [15] takes advantage of the three-term recursion for the Chebyshev polynomials and generates the Runge-Kutta-Chebyshev (RKC) family of order $p=1$ (see also [3, chapter IV.2] or [9, chapter V]).

\section{Runge-Kutta methods seen as regularization schemes}

A Runge-Kutta method (2.2) applied to the autonomous ODE (1.5), that is, $\Psi(t, w(t))=$ $T^{*}(y-T w(t))$ in $(2.1)$, reduces to

$$
\begin{array}{ll}
w_{n}=w_{n-1}+\Delta \mathrm{t}_{n} \sum_{i=1}^{s} b_{i} k_{i}\left(w_{n-1}, \Delta \mathrm{t}_{n}\right), & w_{0}=0, \\
k_{i}+\Delta \mathrm{t}_{n} \sum_{j=1}^{s} a_{i j} T^{*} T k_{j}=T^{*}\left(y-T w_{n-1}\right), & i=1, \ldots, s .
\end{array}
$$

The above system of equations determining the stages $k_{i}$ is well-posed for appropriate $\Delta \mathrm{t}_{n}$ as we show in theorems 3.2 and 3.3. A numerical implementation will be addressed in section 4.1.

In view of (2.3) we may also write

$$
w_{n}=R\left(-\Delta \mathrm{t}_{n} T^{*} T\right) w_{n-1}+\Delta \mathrm{t}_{n} Q\left(-\Delta \mathrm{t}_{n} T^{*} T\right) T^{*} y, \quad w_{0}=0,
$$

2 SDIRK: singly diagonally implicit Runge-Kutta. 
and further, by resolving the recursion, we find that

$$
w_{n}=\mathcal{R}_{n} y=F_{n}\left(T^{*} T\right) T^{*} y
$$

with

$$
F_{n}(\lambda)=\sum_{j=1}^{n}\left(\prod_{k=j+1}^{n} R\left(-\Delta \mathrm{t}_{k} \lambda\right)\right) \Delta \mathrm{t}_{j} Q\left(-\Delta \mathrm{t}_{j} \lambda\right) .
$$

Lemma 3.1. We have that

$$
F_{n}(\lambda)=\frac{1-\prod_{k=1}^{n} R\left(-\Delta \mathrm{t}_{k} \lambda\right)}{\lambda} .
$$

Proof. Taking into account that $Q(z)=(R(z)-1) / z$ the stated representation of $F_{n}$ is straightforwardly established by an inductive argument with respect to $n$.

Now we will validate that the family $\left\{F_{n}\right\}_{n \in \mathbb{N}_{0}}$ is a filter with infinite qualification.

Theorem 3.2. Let $T$ be in $\mathcal{L}(X, Y)$ and let $R$ be the stability function of a Runge-Kutta method of order 1 at least ${ }^{3}$.

Then, there is a maximal step size $\Delta \mathrm{t}_{\max }>0$ such that for any positive $\Delta \mathrm{t}_{\min }<\Delta \mathrm{t}_{\max }$ the family $\left\{F_{n}\right\}_{n \in \mathbb{N}_{0}}$ defined in (3.2) with $\left\{\Delta \mathrm{t}_{n}\right\}_{n \in \mathbb{N}} \subset\left[\Delta \mathrm{t}_{\min }, \Delta \mathrm{t}_{\max }[\right.$ satisfies (1.2) as well as (1.4) for any $\mu>0$, that is, $\mu_{\mathrm{Q}}=\infty$.

In other words, Runge-Kutta integrators with step sizes small enough and bounded away from zero are regularization schemes of optimal order in $\mathrm{X}_{\mu, \varrho}$ for all $\mu, \varrho>0$ when stopped by the discrepancy principle.

Proof. Due to the order 1 of the Runge-Kutta integrator we have the Taylor expansion

$$
R(z)=1+z+\mathrm{O}\left(z^{2}\right) \quad \text { as } \quad z \rightarrow 0 .
$$

Therefore, there exists a positive $z_{\max }$ such that for any positive $z_{\min }<z_{\max }$ there is a bound $\mathbf{r}=\mathbf{r}\left(z_{\min }\right)<1$ for which

$$
|R(-z)| \leqslant \mathbf{r}\left(z_{\min }\right)<1 \quad \text { uniformly in } z \in\left[z_{\min }, z_{\max }\right] .
$$

Observe that $\mathbf{r}\left(z_{\min }\right) \rightarrow 1$ from below as $z_{\min } \rightarrow 0$. Define

$$
\Delta \mathrm{t}_{\max }:=z_{\max } /\|T\|^{2}
$$

and choose $\Delta \mathrm{t}_{\min }<\Delta \mathrm{t}_{\max }$. Let $\left\{\Delta \mathrm{t}_{n}\right\}_{n \in \mathbb{N}} \subset\left[\Delta \mathrm{t}_{\min }, \Delta \mathrm{t}_{\max }[\right.$ and let $\left.\lambda \in] 0,\|T\|^{2}\right]$. Then, $\left|R\left(-\Delta \mathrm{t}_{n} \lambda\right)\right| \leqslant \mathbf{r}\left(\Delta \mathrm{t}_{\min } \lambda\right)$ uniformly in $n$. Hence,

$$
\lim _{n \rightarrow \infty} F_{n}(\lambda)=1 / \lambda
$$

as well as

$$
\lambda\left|F_{n}(\lambda)\right|=1-\prod_{k=1}^{n} R\left(-\Delta \mathrm{t}_{k} \lambda\right) \leqslant 2,
$$

that is, $\left\{F_{n}\right\}_{n \in \mathbb{N}_{0}}$ fulfils (1.2).

Now, we determine the qualification of the filter. Below we will verify the existence of a positive $x_{R}$ such that

$$
R(-x)^{2} \leqslant \mathrm{e}^{-x} \quad \text { for all } \quad x \in\left[0, x_{R}\right] .
$$

3 Any meaningful Runge-Kutta scheme has order 1 at least since order 1 is equivalent to the consistency of the method with the underlying ODE. The condition $\sum_{i=1}^{s} b_{i}=1$ guarantees order 1 . 
For the time being assume the above inequality to hold true. Recall that $\left|1-\lambda F_{n}(\lambda)\right|=$ $\prod_{k=1}^{n}\left|R\left(-\Delta \mathrm{t}_{k} \lambda\right)\right|$. Let $\mu>0$ and define $t_{n}:=\sum_{k=1}^{n} \Delta \mathrm{t}_{k}, \vartheta:=t_{n} \lambda$ as well as $h(\vartheta):=\vartheta^{\mu / 2} \prod_{k=1}^{n}\left|R\left(-\Delta \mathrm{t}_{k} \vartheta / t_{n}\right)\right|$. Then,

$$
\sup _{0 \leqslant \lambda \leqslant\|T\|^{2}} \lambda^{\mu / 2}\left|1-\lambda F_{n}(\lambda)\right|=t_{n}^{-\mu / 2} \sup _{0 \leqslant \vartheta \leqslant t_{n}\|T\|^{2}} h(\vartheta) .
$$

First, we consider $\vartheta \in\left[0, t_{n} x_{R} / \Delta \mathrm{t}_{\max }\right]$ which gives $0 \leqslant \Delta \mathrm{t}_{k} \vartheta / t_{n} \leqslant x_{R}$. Here, we have

$$
\begin{aligned}
& \sup _{0 \leqslant \vartheta \leqslant t_{n} x_{R} / \Delta \mathrm{t}_{\max }} h(\vartheta)=\sup _{0 \leqslant \vartheta \leqslant t_{n} x_{R} / \Delta \mathrm{t}_{\max }} \vartheta^{\mu / 2}\left(\prod_{k=1}^{n}\left|R\left(-\Delta \mathrm{t}_{k} \vartheta / t_{n}\right)\right|^{2}\right)^{1 / 2} \\
& \stackrel{(3.5)}{\leqslant} \sup _{0 \leqslant \vartheta \leqslant t_{n} x_{R} / \Delta \mathrm{t}_{\max }} \vartheta^{\mu / 2} \mathrm{e}^{-\vartheta / 2} \leqslant\left(\frac{\mu}{\mathrm{e}}\right)^{\mu / 2} \text {. }
\end{aligned}
$$

If $\|T\|^{2} \leqslant x_{R} / \Delta \mathrm{t}_{\max }$ we are done. Otherwise, we inspect $\left.\left.\vartheta \in\right] t_{n} x_{R} / \Delta \mathrm{t}_{\max }, t_{n}\|T\|^{2}\right]$. As $\left\{\Delta \mathrm{t}_{n}\right\}_{n \in \mathbb{N}} \subset\left[\Delta \mathrm{t}_{\min }, \Delta \mathrm{t}_{\max }\right.$ [we find that $\underline{x}:=\Delta \mathrm{t}_{\min } x_{R} / \Delta \mathrm{t}_{\max }<\Delta \mathrm{t}_{k} \vartheta / t_{n}<z_{\max }$. Thus,

$\sup _{t_{n} x_{R} / \Delta \mathrm{t}_{\max } \leqslant \vartheta \leqslant t_{n}\|T\|^{2}} h(\vartheta) \leqslant \sup _{t_{n} x_{R} / \Delta \mathrm{t}_{\max } \leqslant \vartheta \leqslant t_{n}\|T\|^{2}} \vartheta^{\mu / 2} \mathbf{r}(\underline{x})^{n} \leqslant\|T\|^{\mu} \sup _{n \in \mathbb{N}} t_{n}^{\mu / 2} \mathbf{r}(\underline{x})^{n}$.

The supremum on the right is finite since $t_{n}<n \Delta \mathrm{t}_{\max }$. Collecting the pieces we end up with

$$
\sup _{0 \leqslant \lambda \leqslant\|T\|^{2}} \lambda^{\mu / 2}\left|1-\lambda F_{n}(\lambda)\right| \leqslant C_{Q} t_{n}^{-\mu / 2} \quad \text { for any } \quad n \in \mathbb{N}
$$

where

$$
C_{Q}=\max \left\{\left(\frac{\mu}{\mathrm{e}}\right)^{\mu / 2},\|T\|^{\mu} \sup _{n \in \mathbb{N}} t_{n}^{\mu / 2} \mathbf{r}(\underline{x})^{n}\right\}
$$

Since $|R(-z)| \leqslant 1$ for $z \in\left[0, z_{\max }\right]$, both functions $R(-z)$ and $Q(-z)$ are continuous there. Hence, $K=\sup \left\{|Q(-z)|: 0 \leqslant z \leqslant z_{\max }\right\}<\infty$ and

$$
\sup \left\{\left|F_{n}(\lambda)\right|: 0 \leqslant \lambda \leqslant\|T\|^{2}\right\} \stackrel{3.2}{\leqslant} K \sum_{j=1}^{n} \Delta \mathrm{t}_{j}=K t_{n}
$$

for any $n \in \mathbb{N}_{0}$. The two estimates (3.6) and (3.7) reveal finally the infinite qualification of the Runge-Kutta filter $\left\{F_{n}\right\}_{n \in \mathbb{N}_{0}}$.

We are not finished yet with the proof of theorem 3.2. It remains to establish (3.5). From the Taylor expansion (3.3) we see that $R(0)=R^{\prime}(0)=1$. Therefore, $R(-x)$ is positive for $x$ sufficiently small. Further, for $r(x)=R(-x)^{2}$ we obtain $r(0)=1$ and $r^{\prime}(0)=-2 R(0) R^{\prime}(0)=-2$. Consequently, the difference $d(x)=r(x)-\mathrm{e}^{-x}$ satisfies $d(0)=0$ and $d^{\prime}(0)=-1$ which yields the estimate on the right in (3.5) and completes the proof of theorem 3.2.

We have no restriction on the maximal time step, that is, any finite $\Delta \mathrm{t}_{\max }$ is admissible in theorem 3.2, whenever

$$
|R(-z)|<1 \quad \text { for all } z>0 .
$$

In this respect A-stable methods satisfying (3.8) generate regularization schemes which are convenient to use as no information on the magnitude of $\|T\|$ is required and $\Delta \mathrm{t}_{\max }$ can be chosen arbitrarily large. Strongly A-stable methods with (3.8) are even more attractive because $z_{\max }=\infty$ is admissible in (3.4). Therefore the convergence does not deteriorate for large time steps. 
Theorem 3.3. Let $T$ be in $\mathcal{L}(X, Y)$ and let $R$ be the stability function of an A-stable RungeKutta method which satisfies (3.8). Let the time steps $\left\{\Delta \mathrm{t}_{n}\right\}_{n \in \mathbb{N}}$ be bounded from below by $\Delta \mathrm{t}_{\min }>0$ and from above by $\Delta \mathrm{t}_{\max }$.

Then, the family $\left\{F_{n}\right\}_{n \in \mathbb{N}_{0}}$ defined in (3.2) satisfies (1.2) as well as (1.4) for any $\mu>0$, that is, $\mu_{\mathrm{Q}}=\infty$.

In other words, A-stable Runge-Kutta integrators with step sizes bounded from above and bounded away from zero are regularization schemes of optimal order in $\mathrm{X}_{\mu, \varrho}$ for all $\mu, \varrho>0$ when stopped by the discrepancy principle.

Proof. Since (3.4) holds for $z_{\max }=\Delta \mathrm{t}_{\max } /\|T\|^{2}$ we obtain, as in the proof of theorem 3.2, that the filter $\left\{F_{n}\right\}_{n \in \mathbb{N}_{0}}$ fulfils (1.2). Further, we can find an $m \in \mathbb{N}, m \geqslant 2$, and an $x_{R, m}>0$ such that

$\|T\|^{2} \leqslant x_{R, m} / \Delta \mathrm{t}_{\max } \quad$ and $\quad|R(-x)|^{m} \leqslant \mathrm{e}^{-x} \quad$ for all $\quad x \in\left[0, x_{R, m}\right]$.

We will verify (3.9) at the end of this proof.

Let $t_{n}$ and $h(\vartheta)$ be defined as in the proof of theorem 3.2. Then,

$$
\begin{aligned}
\sup _{0 \leqslant \lambda \leqslant\|T\|^{2}} \lambda^{\mu / 2}\left|1-\lambda F_{n}(\lambda)\right| & =t_{n}^{-\mu / 2} \sup _{0 \leqslant \vartheta \leqslant t_{n}\|T\|^{2}} h(\vartheta) \\
& \leqslant t_{n}^{-\mu / 2} \sup _{0 \leqslant \vartheta \leqslant t_{n} x_{R, m} / \Delta \mathrm{t}_{\max }} h(\vartheta) \\
& =t_{n}^{-\mu / 2} \sup _{0 \leqslant \vartheta \leqslant t_{n} x_{R, m} / \Delta \mathrm{t}_{\max }} \vartheta^{\mu / 2}\left(\prod_{k=1}^{n}\left|R\left(-\Delta \mathrm{t}_{k} \vartheta / t_{n}\right)\right|^{m}\right)^{1 / m} \\
& \stackrel{(3.9)}{\leqslant} t_{n}^{-\mu / 2} \sup _{0 \leqslant \vartheta<\infty} \vartheta^{\mu / 2} \mathrm{e}^{-\vartheta / m}=t_{n}^{-\mu / 2}\left(\frac{m \mu}{2 \mathrm{e}}\right)^{\mu / 2} .
\end{aligned}
$$

Additionally (3.7) holds with $K=\sup \{|Q(-z)|: z \in[0, \infty[\}<\infty$. Thus, we have the infinite qualification of $\left\{F_{n}\right\}_{n \in \mathbb{N}_{0}}$.

For the proof of (3.9) it suffices to construct a strongly monotone increasing, unbounded sequence $\left.\left\{x_{R, k}\right\}_{k \geqslant 2} \subset\right] 0, \infty[$ such that

$|R(-x)|^{k} \leqslant \mathrm{e}^{-x} \quad$ for all $\quad x \in\left[0, x_{R, k}\right] \quad$ and $\quad\left|R\left(-x_{R, k}\right)\right|^{k}=\mathrm{e}^{-x_{R, k}}$.

We construct $\left\{x_{R, k}\right\}_{k \geqslant 2}$ recursively starting with $x_{R, 2}=x_{R}$ where $x_{R}$ is from (3.5). Now let $x_{R, k-1}$ be known. By

$$
\left|R\left(-x_{R, k-1}\right)\right|^{k}=\left|R\left(-x_{R, k-1}\right)\right| \mathrm{e}^{-x_{R, k-1} \stackrel{(3.8)}{<}} \mathrm{e}^{-x_{R, k-1}}
$$

there exists an $x_{R, k}>x_{R, k-1}$ for which (3.10) holds (recall that $R$ is a rational function). The constructed sequence $\left\{x_{R, k}\right\}$ grows unbounded. Indeed, assume the contrary. Then, $\left\{x_{R, k}\right\}$ converges, say, to $x_{R, \infty}<\infty$. Since $\left|R\left(-x_{R, k}\right)\right|=\mathrm{e}^{-x_{R, k} / k}$ for all $k \geqslant 2$ we find that $\left|R\left(-x_{R, \infty}\right)\right|=1$ which contradicts (3.8).

The four Runge-Kutta integrators from example 2.1 induce the following iterative regularization schemes.

Example 3.4. The implicit Euler scheme with $R(z)=1 /(1-z)$ and $Q(z)=R(z)$ yields the iteration

$$
\begin{aligned}
w_{n} & =\left(I+\Delta \mathrm{t}_{n} T^{*} T\right)^{-1} w_{n-1}+\Delta \mathrm{t}_{n}\left(I+\Delta \mathrm{t}_{n} T^{*} T\right)^{-1} T^{*} y \\
& =\left(I+\Delta \mathrm{t}_{n} T^{*} T\right)^{-1}\left(w_{n-1}+\Delta \mathrm{t}_{n} T^{*} y\right)
\end{aligned}
$$


which is also known as nonstationary iterated Tikhonov-Phillips regularization. Since (3.8) and L-stability hold, theorem 3.3 applies. Several authors have investigated the nonstationary iterated Tikhonov-Phillips regularization before, for instance, Schock [13] and Hanke and Groetsch [4]. Theorem 3 from [4], which only applies to the nonstationary iterated TikhonovPhillips regularization, is slightly more general than our theorem 3.3 insofar as geometrically growing sequences $\left\{\Delta \mathrm{t}_{n}\right\}$ and unbounded operators are covered.

Fast growing step sizes are attractive because they determine the convergence speed in the case of unperturbed data:

$$
\left\|f^{+}-\mathcal{R}_{n} T f^{+}\right\|_{X} \leqslant C_{Q} \varrho\left(\sum_{j=1}^{n} \Delta \mathrm{t}_{j}\right)^{-\mu / 2} \quad \text { for any } \quad f^{+} \in \mathrm{X}_{\mu, \rho}
$$

which follows from (3.6) by standard arguments (see, e.g., [2, 11]). In the presence of noise, though, large step sizes may cause trouble (see the comments in section 4.2).

Example 3.5. The SDIRK2 method with $R(z)=(1+(1-2 \alpha) z) /(1-\alpha z)^{2}$ and $Q(z)=\left(1-\alpha^{2} z\right) /(1-\alpha z)^{2}$ yields the iteration

$w_{n}=\left(I+\alpha \Delta \mathrm{t}_{n} T^{*} T\right)^{-2}\left(\left(I-(1-2 \alpha) \Delta \mathrm{t}_{n} T^{*} T\right) w_{n-1}+\Delta \mathrm{t}_{n}\left(I+\alpha^{2} \Delta \mathrm{t}_{n} T^{*} T\right) T^{*} y\right)$.

With $\alpha$ restricted to the interval $[1-\sqrt{2} / 2,1+\sqrt{2} / 2]$ the SDIRK2 method is L-stable and (3.8) holds. Hence, theorem 3.3 applies.

Example 3.6. The explicit Euler scheme with $R(z)=1+z$ and $Q(z)=1$ yields the iteration

$$
w_{n}=\left(I-\Delta \mathbf{t}_{n} T^{*} T\right) w_{n-1}+\Delta \mathbf{t}_{n} T^{*} y=w_{n-1}+\Delta \mathbf{t}_{n} T^{*}\left(y-T w_{n-1}\right)
$$

where $\Delta \mathrm{t}_{\max }=2 /\|T\|^{2}$ is the maximal step size. Thus, we have rediscovered the Landweber iteration (see, e.g., [2, chapter 6.1] and [11, Kapitel 5.1]).

Example 3.7. The RKCs method ${ }^{4}$ with $R(z)=\mathrm{T}_{s}\left(\omega_{0}+\omega_{1} z\right) / \mathrm{T}_{s}\left(\omega_{0}\right), \omega_{1}=\mathrm{T}_{s}\left(\omega_{0}\right) / \mathrm{T}_{s}^{\prime}\left(\omega_{0}\right)$, generates the iteration (see appendix A)

$$
\begin{aligned}
& w_{n, 0}=w_{n-1}, \quad w_{n, 1}=w_{n, 0}+\tilde{\mu}_{1} \Delta \mathrm{t}_{n} T^{*}\left(y-T w_{n, 0}\right), \\
& \text { for } j=2, \ldots, s \\
& w_{n, j}=\mu_{j} w_{n, j-1}+v_{j} w_{n, j-2}+\tilde{\mu}_{j} \Delta \mathrm{t}_{n} T^{*}\left(y-T w_{n, j-1}\right), \\
& w_{n}=w_{n, s},
\end{aligned}
$$

where $\Delta \mathrm{t}_{\max }=2 \omega_{0} / \omega_{1} /\|T\|^{2}$ and where, for $j=2, \ldots, s$,

$$
\begin{array}{ll}
\mu_{j}=\frac{2 \omega_{0} \mathrm{~T}_{j-1}\left(\omega_{0}\right)}{\mathrm{T}_{j}\left(\omega_{0}\right)}, & v_{j}=-\frac{\mathrm{T}_{j-2}\left(\omega_{0}\right)}{\mathrm{T}_{j}\left(\omega_{0}\right)}, \\
\tilde{\mu}_{1}=\frac{\omega_{1}}{\omega_{0}}=\frac{\mathrm{T}_{s}\left(\omega_{0}\right)}{\omega_{0} \mathrm{~T}_{s}^{\prime}\left(\omega_{0}\right)}, & \tilde{\mu}_{j}=\frac{2 \omega_{1} \mathrm{~T}_{j-1}\left(\omega_{0}\right)}{\mathrm{T}_{j}\left(\omega_{0}\right)} .
\end{array}
$$

Please observe that $\mathrm{RKC} 1$ is identical to the Landweber iteration. From this point of view the RKCs methods are generalizations of the Landweber iteration.

Remark 3.8. For the linear problems considered so far only the stability function $R$ determines the iterative regularization scheme (3.1). Thus, different Runge-Kutta schemes may yield the same iterative regularization. For nonlinear problems (see section 6.2), however, the Butcher array, that is, the succession of the stages, comes into play crucially.

${ }^{4}$ RKCs method: Runge-Kutta-Chebyshev method of $s$ stages and order $p=1$. 


\section{Numerical experiments}

The Runge-Kutta regularization schemes considered as examples in the former section shall be illustrated and compared by numerical experiments.

We are going to solve the linear integral equation

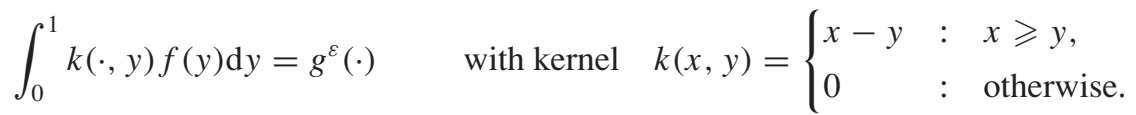

The integral operator $T f(\cdot):=\int_{0}^{1} k(\cdot, y) f(y) \mathrm{d} y$ maps $L^{2}(0,1)$ compactly into itself. The equality $T f=g$ holds true iff $g^{\prime \prime}=f$ and $g(0)=g^{\prime}(0)=0$. Therefore, $\mathrm{N}(T)=\{0\}$.

\subsection{Discretization}

We discretize the integral equation using a projection method. As finite-dimensional approximation space $X_{l}$ of $L^{2}(0,1)$ we choose the space of continuous piecewise linear functions with respect to the step size $h_{l}=1 /(l-1), l \in \mathbb{N}, l \geqslant 2$. Note that $X_{l}$ is spanned by the hat functions $\varphi_{l, j} \in X_{l}, j=1, \ldots, l$, which are characterized by

$$
\varphi_{l, j}\left((i-1) h_{l}\right):=\left\{\begin{array}{lll}
1 & : & i=j, \\
0 & : & \text { otherwise, }
\end{array} \quad i=1, \ldots, l .\right.
$$

Let $P_{l}: L^{2}(0,1) \rightarrow L^{2}(0,1)$ be the orthogonal projector onto $X_{l}$ and set $T_{l}:=P_{l} T P_{l}$. Now we are seeking a stable approximation to $f^{+}$starting from the finite-dimensional system

$$
T_{l} f_{l}=P_{l} g^{\varepsilon} .
$$

To this end we apply a Runge-Kutta scheme to the above equation yielding

$$
\mathcal{R}_{l, n} g^{\varepsilon}=F_{n}\left(T_{l}^{*} T_{l}\right) T_{l}^{*} P_{l} g^{\varepsilon}
$$

with the filter $F_{n}$ from (3.2). As a stopping rule we work with the modified discrepancy principle (4.3) below taking discretization effects into account: let $\zeta_{l} \in \mathbb{R}$ satisfy $\left\|T\left(I-P_{l}\right)\right\| \leqslant$ $\zeta_{l}$ and choose $\tau>1$. Define

$$
\mathcal{D}_{l}=\left\{k \in \mathbb{N}_{0}:\left\|T_{l} \mathcal{R}_{l, k} g^{\varepsilon}-P_{l} g^{\varepsilon}\right\|_{L^{2}(0,1)} \leqslant \tau \varepsilon \text { and } k \leqslant \zeta_{l}^{-2}\right\}
$$

and determine

$$
\gamma\left(\varepsilon, g^{\varepsilon}, l\right)= \begin{cases}\min \mathcal{D}_{l} & : \mathcal{D}_{l} \neq \emptyset \\ \min \left\{k \in \mathbb{N}_{0}: k>\zeta_{l}^{-2}\right\} & : \quad \text { otherwise. }\end{cases}
$$

The modified discrepancy principle reduces to Morozov's discrepancy principle (1.3) in the case when no discretization is performed, that is, $P_{l}=I$ and $\zeta_{l}=0$. The following error estimate is essentially due to Plato [10] (see also [11, Kapitel 6.2]).

Theorem 4.1. Let the Runge-Kutta scheme and the chosen step sizes $\left\{\Delta \mathrm{t}_{n}\right\}_{n \in \mathbb{N}}$ in (4.2) satisfy either the hypotheses of theorem 3.2 or of theorem 3.3.

If $f^{+} \in \mathrm{X}_{\mu, \varrho}, \mu, \varrho>0$, and if $\left\|T f^{+}-g^{\varepsilon}\right\|_{L^{2}(0,1)} \leqslant \varepsilon$ then there is a constant $\widetilde{C}_{\mu}$ such that

$$
\left\|f^{+}-\mathcal{R}_{l, \gamma\left(\varepsilon, g^{\varepsilon}, l\right)} g^{\varepsilon}\right\|_{L^{2}(0,1)} \leqslant \widetilde{C}_{\mu}\left(\varepsilon^{\mu /(\mu+1)} \varrho^{1 /(\mu+1)}+\varrho\left(\zeta_{l}^{\min \{\mu, 1\}}+\left\|\left(I-P_{l}\right) T\right\|^{\min \{\mu, 2\}}\right)\right)
$$

For the numerical computation we need a matrix-based version of (4.2). Therefore we introduce the matrices $\mathbf{T}_{l}, \mathbf{G}_{l} \in \mathbb{R}^{l \times l}$ with entries

$$
\left(\mathbf{T}_{l}\right)_{i, j}=\left\langle\varphi_{l, i}, T \varphi_{l, j}\right\rangle_{L^{2}(0,1)} \quad \text { and } \quad\left(\mathbf{G}_{l}\right)_{i, j}=\left\langle\varphi_{l, i}, \varphi_{l, j}\right\rangle_{L^{2}(0,1)}
$$


which will be computed analytically for our experiments below. We have that $\mathcal{R}_{l, n} g^{\varepsilon}=$ $\sum_{i=1}^{l}\left(w_{l, n}\right)_{i} \varphi_{l, i}$ iff ${ }^{5}$

$w_{l, n}=F_{n}\left(\mathbf{G}_{l}^{-1} \mathbf{T}_{l}^{t} \mathbf{G}_{l}^{-1} \mathbf{T}_{l}\right) \mathbf{G}_{l}^{-1} \mathbf{T}_{l}^{t} \mathbf{G}_{l}^{-1} q_{l}\left(g^{\varepsilon}\right) \quad$ with $\quad q_{l}\left(g^{\varepsilon}\right)=\left(\begin{array}{c}\left\langle\varphi_{l, 1}, g^{\varepsilon}\right\rangle_{L^{2}} \\ \vdots \\ \left\langle\varphi_{l, l}, g^{\varepsilon}\right\rangle_{L^{2}}\end{array}\right)$,

see [11, Kapitel 6.1.1]. For instance, the implicit Euler scheme (example 3.4) yields the iteration

$$
w_{l, n}=\left(\mathbf{I}+\Delta \mathbf{t}_{n} \mathbf{G}_{l}^{-1} \mathbf{T}_{l}^{t} \mathbf{G}_{l}^{-1} \mathbf{T}_{l}\right)^{-1}\left(w_{l, n-1}+\Delta \mathbf{t}_{n} \mathbf{G}_{l}^{-1} \mathbf{T}_{l}^{t} \mathbf{G}_{l}^{-1} q_{l}\left(g^{\varepsilon}\right)\right) .
$$

Remark 4.2. Constant time steps $\Delta \mathrm{t}_{n}=\Delta \mathrm{t}$ are especially attractive for implicit methods from a computational point of view as the matrix of the linear system, which has to be solved in each iteration step, does not depend on $n$. Thus, a LU decomposition of the matrix can be computed in advance reducing the numerical effort.

\subsection{Computations}

In our first experiments we want to illustrate the error estimate from theorem 4.1 for $\mu \in\{1,2\}$. We work with the two different exact solutions $f_{1}^{+}=T^{*} w_{1}, w_{1}(x)=2-9 \pi^{2} \cos (3 \pi x) / 2$ (example 1) and $f_{2}^{+}=T^{*} T w_{2}, w_{2}=1$ (example 2). Thus, $f_{\mu}^{+} \in \mathrm{R}\left(|T|^{\mu}\right), \mu \in\{1,2\}$. The corresponding right-hand sides are $g_{1}(x)=x^{2}\left(x^{2}-4 x+9\right) / 12+(1-\cos (3 \pi x)) / \pi^{2} / 18$ and $g_{2}(x)=x^{2}\left(x^{4} / 720-x / 36+1 / 16\right)$.

To set up the discrete schemes we have to evaluate $q_{l}\left(g^{\varepsilon}\right)$, that is, we have to compute the inner products $\left\langle\varphi_{l, i}, g^{\varepsilon}\right\rangle_{L^{2}}$. These integrals cannot be determined analytically in general. We circumvent the problem by working with a discrete right-hand side $g^{\varepsilon}$ obtained from $g$ via interpolation and perturbation $\left(x_{l, j}:=(j-1) h_{l}\right)$ :

$$
g^{\varepsilon}:=\sum_{j=1}^{l}\left(g\left(x_{l, j}\right)+\delta\left(x_{l, j}\right)\right) \varphi_{l, j}
$$

where $\delta:[0,1] \rightarrow[-\delta, \delta], \delta>0$, denotes a uniformly distributed random variable. Now we have

$$
q_{l}\left(g^{\varepsilon}\right)=\mathbf{G}_{l} \mathbf{g} \quad \text { with } \quad \mathbf{g}=\left(g^{\varepsilon}\left(x_{l, 1}\right), \ldots, g^{\varepsilon}\left(x_{l, l}\right)\right)^{t} .
$$

The noise level in $g^{\varepsilon}$ can be estimated by

$$
\left\|g-g^{\varepsilon}\right\|_{L^{2}(0,1)} \leqslant h_{l}^{2}\left\|g^{\prime \prime}\right\|_{L^{2}(0,1)}+\delta=: \varepsilon .
$$

Moreover,

$$
\left\|T\left(I-P_{l}\right)\right\| \leqslant h_{l}^{2} \quad \text { as well as } \quad\left\|\left(I-P_{l}\right) T\right\| \leqslant h_{l}^{2} .
$$

The last three estimates are verified in [11, Kapitel 6.2.3]. Choosing $\delta=h_{l}^{2}$ we therefore expect to observe the following error behaviour according to theorem 4.1:

$$
\left\|f_{\mu}^{+}-\mathcal{R}_{l, \gamma\left(\varepsilon, g_{\mu}^{\varepsilon}, l\right)} g_{\mu}^{\varepsilon}\right\|_{L^{2}(0,1)}=\mathrm{O}\left(h_{l}^{2 \mu /(\mu+1)}\right) \quad \text { as } \quad l \rightarrow \infty, \quad \mu \in\{1,2\} .
$$

In principle we are able to determine the numerical value of the reconstruction error $\left\|f_{\mu}^{+}-\mathcal{R}_{l, \gamma\left(\varepsilon, g_{\mu}^{\varepsilon}, l\right)} g_{\mu}^{\varepsilon}\right\|_{L^{2}}$ exactly. However, we can rely on the trapezoidal rule $\operatorname{TR}_{h_{l}}(v)$ with respect to the step size $h_{l}$ for evaluating the integral $\int_{0}^{1} v(t) \mathrm{d} t$. Indeed,

$$
\operatorname{Err}(l):=\left(\operatorname{TR}_{h_{l}}\left(\left(f_{\mu}^{+}-\mathcal{R}_{l, \gamma\left(\varepsilon, g_{\mu}^{\varepsilon}, l\right)} g_{\mu}^{\varepsilon}\right)^{2}\right) / \operatorname{TR}_{h_{l}}\left(\left(f_{\mu}^{+}\right)^{2}\right)\right)^{1 / 2}
$$

5 As $F_{n}$ is a rational function the evaluation of $F_{n}\left(\mathbf{G}_{l}^{-1} \mathbf{T}_{l}^{t} \mathbf{G}_{l}^{-1} \mathbf{T}_{l}\right)$ is well defined (see, e.g., [11, lemma 6.2] for further details). 


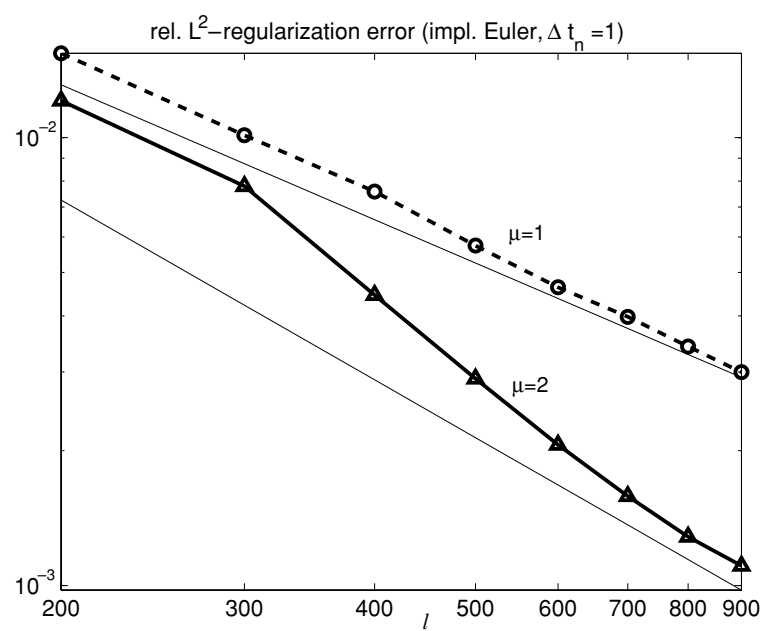

Figure 1. Relative regularization error Err (4.5) as a function of $l$ using the implicit Euler scheme with $\Delta \mathrm{t}=1$ (solid line with $\triangle$ : example 2, dashed line with $\circ$ : example 1$)$. The thin solid lines indicate decay $\mathrm{O}\left(h_{l}\right)$ and $\mathrm{O}\left(h_{l}^{4 / 3}\right)$ as $l \rightarrow \infty$.

approximates the relative $L^{2}$-regularization error with order $h_{l}^{2}$. Thus,

$$
\operatorname{Err}(l)=\mathrm{O}\left(h_{l}^{2 \mu /(\mu+1)}\right) \quad \text { as } \quad l \rightarrow \infty .
$$

The expected asymptotic behaviour can be observed by numerical computations. In figure 1 the relative error Err is plotted obtained by the implicit Euler scheme with constant time step size $\Delta \mathrm{t}=1$.

Next we will compare different Runge-Kutta schemes in both reconstruction accuracy and run-time. Here, the choice of the time steps $\left\{\Delta t_{n}\right\}_{n}$ is crucial for meaningful and just results. The step size control procedures very successfully implemented for solving ODEs are unsuitable in the present context because we are not interested in solving the evolution equation (1.5) accurately for long times. Rather we want to get close to the stationary solution of (1.5) as fast as possible. Accordingly we wish to work with large time steps, compare (3.11). On the other hand, if we select the time steps too large then the reconstruction $\mathcal{R}_{l, \gamma\left(\varepsilon, g^{\varepsilon}, l\right)} g^{\varepsilon}$ might over-satisfy the discrepancy principle, that is, $\left\|T_{l} \mathcal{R}_{l, \gamma\left(\varepsilon, g^{\varepsilon}, l\right)} g^{\varepsilon}-P_{l} g^{\varepsilon}\right\|_{L^{2}} \ll \tau \varepsilon$. Thus the noise in $g^{\varepsilon}$ gets amplified and the reconstruction is useless in spite of a small discrepancy. To avoid noise amplification we recommend monitoring the quotient

$$
q_{l}:=\frac{\left\|T_{l} \mathcal{R}_{l, \gamma\left(\varepsilon, g^{\varepsilon}, l\right)} g^{\varepsilon}-P_{l} g^{\varepsilon}\right\|_{L^{2}}}{\tau \varepsilon}
$$

and accepting $\mathcal{R}_{l, \gamma\left(\varepsilon, g^{\varepsilon}, l\right)} g^{\varepsilon}$ as the approximate solution if $q_{l} \approx 1$. Otherwise, $\mathcal{R}_{l, \gamma\left(\varepsilon, g^{\varepsilon}, l\right)} g^{\varepsilon}$ should be rejected and the last time step should be decreased.

Figure 2 displays the CPU-times ${ }^{6}$ for computing $\mathcal{R}_{l, \gamma\left(\varepsilon, g_{1}^{\varepsilon}, l\right)} g_{1}^{\varepsilon}$ (example 1) by the four schemes: implicit Euler, SDIRK2 with $\alpha=1+\sqrt{2} / 2$, RKC5, and RKC8. All schemes are furnished with constant time steps, that is, $\Delta \mathrm{t}_{n}=\Delta \mathrm{t}$ where $\Delta \mathrm{t}$ depends on $l$ for the implicit schemes. More precisely, for both RKC iterations we set $\omega_{0}=1.01$ and $\Delta \mathrm{t}=2 \omega_{0} / \omega_{1} /\|T\|^{2}$ which is only slightly smaller than the maximal time step $\Delta \mathrm{t}_{\max }=2 \omega_{0} / \omega_{1} /\left\|\mathbf{G}_{l}^{-1} \mathbf{T}_{l}\right\|^{2}(\mathrm{RKC5}$ : $\Delta \mathrm{t} \approx 529$, RKC8: $\Delta \mathrm{t} \approx 1128$ ). The constant time steps for both implicit methods are taken to be $\Delta \mathrm{t}=l^{3 / 2}$. With these time steps we have that $q_{l} \geqslant 0.96$ for all experiments underlying

\footnotetext{
6 The experiments have been carried out under MATLAB 6.5 (R13) on an Intel Pentium 4 processor with 2.6 GHz.
} 


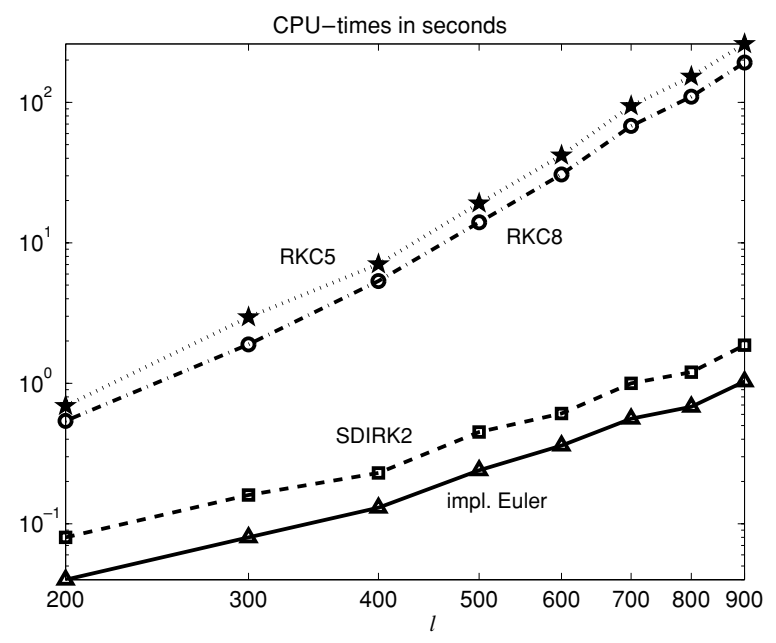

Figure 2. CPU-times for example 1 relative to four integrators stopped by the modified discrepancy principle (4.3): implicit Euler (solid line with $\triangle$ ), SDIRK2 $(\alpha=1+\sqrt{2} / 2$, dashed line with $\square$ ), RKC8 (dashed-dotted line with $\circ$ ), RKC5 (dotted line with $\star$ ). The constant time steps have been adjusted to yield $q_{l} \geqslant 0.96$ for all four integrators.

figure 2. Therefore the measured CPU-times are comparable, the more so because we did not count CPU-time for pre-processing operations shared by all schemes. Moreover, the reconstruction errors (4.5) are virtually identical for all schemes.

As expected the implicit iterations are pronouncedly faster than the explicit ones. In the plotted range for $l$ the CPU-times behave like $\mathrm{O}\left(l^{2}\right)$ and $\mathrm{O}\left(l^{4}\right)$ for the implicit and explicit schemes, respectively ${ }^{7}$. Comparing both implicit integrators we observe that the Euler scheme is twice as fast as the SDIRK2 scheme. While both L-stable methods require about the same number of iterations to be stopped by the discrepancy principle, one iteration step of the SDIRK2 scheme is twice as expensive as one Euler step. Thus, our experiments show that the convergence speed does not necessarily increase with the order of the method. Indeed, the order of a Runge-Kutta integrator tells nothing about convergence speed in the context of regularizing ill-posed problems as we will learn in the next section.

We close the present section by comparing numerically the implicit Euler method with the cg-iteration of Hestenes and Stiefel [5] (cg: conjugate gradients). The cg-iteration stopped by a modified discrepancy principle akin to (4.3) yields a regularization scheme for $T_{l}$, that is, the error estimate of theorem 4.1 holds accordingly (see Plato [10, Satz 5.2] or [11, Satz 6.2.11]). Moreover, the cg-iteration is known to converge rapidly, in fact, under all Krylov subspace methods (like the $v$-methods) the cg-iteration requires the fewest number of iteration steps when the discrepancy principle is the stopping rule.

To obtain the numerical results presented in figure 3 we increased the constant time step of the Euler scheme to $\Delta \mathrm{t}=10 l^{3 / 2}$. With this time step the $q_{l}$ of the Euler scheme are comparable to the $q_{l}$ of the cg-iteration. As a consequence the reconstruction errors (4.5) are almost identical (figure 3 right). Also the CPU-times required by both methods do not differ much although the cg-iteration is slightly faster for large dimensions $l$ (figure 3 left). In our example the numerical effort per iteration step grows like $\mathrm{O}\left(l^{3}\right)$ and $\mathrm{O}\left(l^{2}\right)$ for the implicit Euler integrator and the cg-iteration, respectively. So, the cg-iteration will be

7 For larger $l$ the CPU-times of the implicit schemes grow at least like $\mathrm{O}\left(l^{3}\right)$ since the LU-decomposition, which has to be calculated, dominates the numerical effort. 

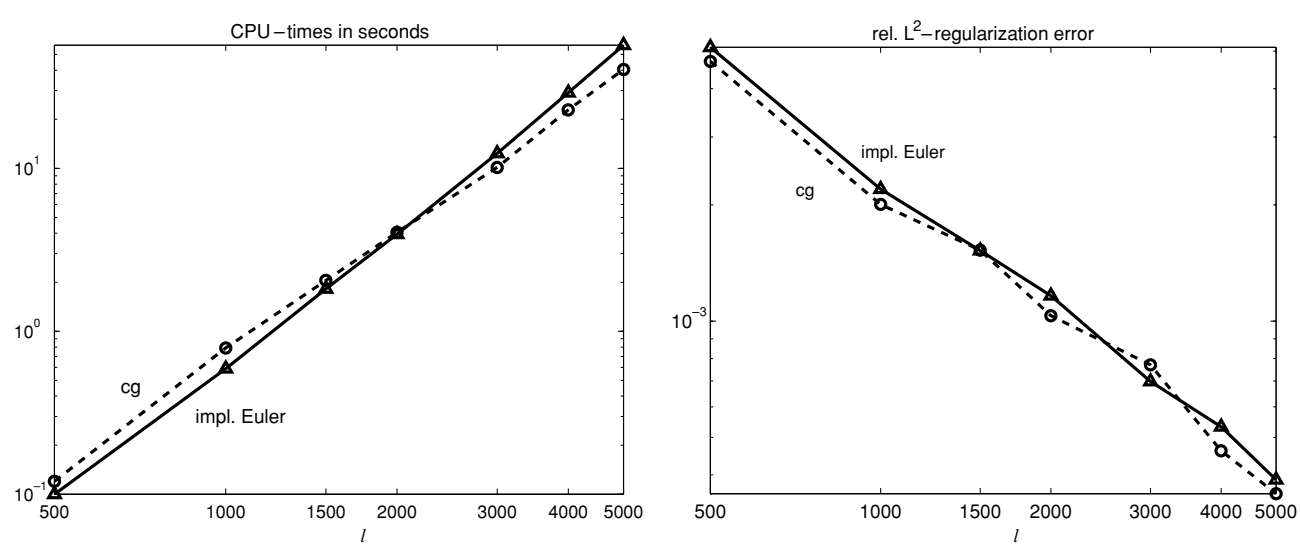

Figure 3. CPU-times and relative $L^{2}$-errors (4.5) for example 1 relative to the cg-iteration (dashed lines with $\circ$ ) and the implicit Euler scheme (solid lines with $\triangle$ ).

faster for large $l$ as long as it requires less than $\mathrm{O}(l)$ iteration steps more than the Euler scheme. Nevertheless, the implicit Euler scheme is an attractive alternative to the cg-iteration which offers an additional parameter, the step size, for fine tuning the stopping criterion to reach $q_{l} \approx 1$. Thus, the implicit Euler scheme combines the advantages of iterative and continuous regularization methods.

\section{Synthetic methods: inconsistent Runge-Kutta schemes}

In the formulations of theorems 3.2 and 3.3 we required the Runge-Kutta scheme to be of order 1 at least. This hypothesis is superfluous however. Indeed, in view of (3.3) the assertions of both theorems remain valid if the stability function of the method has the Taylor expansion

$$
R(z)=1+c z+\mathrm{O}\left(z^{2}\right) \quad \text { as } \quad z \rightarrow 0
$$

where $c>1$. We emphasize that the corresponding Runge-Kutta scheme is inconsistent and therefore useless for solving ODEs.

Can we use the additional freedom (parameter $c$ in (5.1)) to construct schemes which converge faster than the implicit Euler scheme? In a certain sense the answer is 'yes' as we will demonstrate in the remainder of this section.

Since (1.1) is ill posed, zero is a limit point of the spectrum of $T^{*} T$. Thus, as long as $\left.\left.\left|R\left(-z \Delta \mathrm{t}_{\max }\right)\right|<1, z \in\right] 0,\|T\|^{2}\right]$, the eigenvalues close to zero are responsible for the convergence speed. Accordingly, we should construct a method whose stability function $R$ is small for negative arguments close to zero. The latter property can be achieved by requiring $c=R^{\prime}(0) \gg 1$. Altogether we extracted two properties for our desired schemes:

1. $|R(-z)|<1$ for $z>0$ and $|R(\infty)|<1$ (no restriction on the maximal time step, see theorem 3.3),

2. $R^{\prime}(0) \gg 1$ (good damping of contributions of small spectral values).

We present two candidates for potentially outperforming the implicit Euler scheme.

Example 5.1. The Runge-Kutta scheme generated by

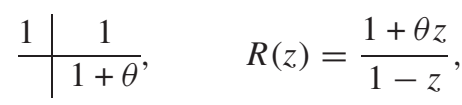


is inconsistent if $\theta \neq 0$. It agrees with the implicit Euler integrator in the consistent case $(\theta=0)$. For $\theta \in[0,1[$ the required properties are fulfilled with $|R(\infty)|=\theta$ and $R^{\prime}(0)=1+\theta$. The corresponding iteration is

$$
w_{n}=\left(I+\Delta \mathrm{t}_{n} T^{*} T\right)^{-1}\left(\left(I-\theta \Delta \mathrm{t}_{n} T^{*} T\right) w_{n-1}+\Delta \mathrm{t}_{n}(1+\theta) T^{*} y\right) .
$$

We call this method SYNTH1 (synthetic method).

In [13] Schock analysed an implicit iterative method of type

$$
w_{n}=\left(\alpha_{n} I+T^{*} T\right)^{-1}\left(\alpha_{n}\left(I-\beta_{n} T^{*} T\right) w_{n-1}+\left(1+\alpha_{n} \beta_{n}\right) T^{*} y\right)
$$

with $\alpha_{n}$ and $\beta_{n}$ properly chosen reals. SYNTH1 coincides with Schock's iteration if $\alpha_{n}=1 / \Delta \mathrm{t}_{n}$ and $\beta_{n}=\theta \Delta \mathrm{t}_{n}$.

Example 5.2. The Butcher array

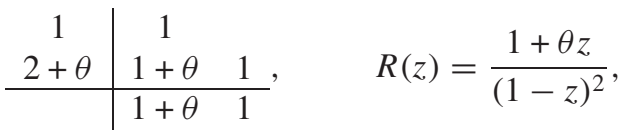

leads to a Runge-Kutta scheme called SYNTH2 which is inconsistent for $\theta \neq-1$. For $\theta=-1$ we have the SDIRK2 method with $\alpha=1$ which reduces to the implicit Euler scheme. The desired properties are satisfied for $\theta \in\left[0,2(1+\sqrt{2})\left[\right.\right.$ with $R^{\prime}(0)=2+\theta$. We even have $|R(\infty)|=0$. The generated iteration reads

$w_{n}=\left(I+\Delta \mathrm{t}_{n} T^{*} T\right)^{-2}\left(\left(I-\theta \Delta \mathrm{t}_{n} T^{*} T\right) w_{n-1}+\Delta \mathrm{t}_{n}\left((2+\theta) I+\Delta \mathrm{t}_{n} T^{*} T\right) T^{*} y\right)$.

Please observe that one step of SYNTH2 coincides with two steps of the implicit Euler scheme when $\theta=0$.

For the comparison of two iterative schemes we would like to decide which stops earlier in general when the discrepancy principle is the stopping rule. To this end we modify an approach by Schock, see [13, Sec. 4] and [12]: Let S1 and S2 be two iterative schemes generating the sequences $\left\{w_{n}^{1}\right\}$ and $\left\{w_{n}^{2}\right\}$, respectively. We say iteration S1 stops (non-uniformly) earlier than iteration $\mathrm{S} 2$ (with respect to the discrepancy principle) if there is a sequence of operators $\left\{Q_{n}\right\} \subset \mathcal{L}(Y)$ converging pointwise to zero such that

$$
T w_{n}^{1}-g^{\varepsilon}=Q_{n}\left(T w_{n}^{2}-g^{\varepsilon}\right) .
$$

Method S1 is said to stop locally (non-uniformly) earlier than method S2 if (5.2) holds true and there is a projection $P$ of finite rank such that $\left\{Q_{n}(I-P)\right\}$ converges pointwise to zero.

Theorem 5.3. Let $T$ be in $\mathcal{L}(X, Y)$ with a non-closed range. We consider SYNTH1 with $0<\theta<1$ and the implicit Euler scheme where both schemes share the same constant step size $\Delta \mathrm{t}>0$.

(a) If $\theta \Delta \mathrm{t}\|T\|^{2}<2$ then SYNTH1 stops earlier than the implicit Euler scheme.

(b) If $\theta \Delta \mathrm{t}\|T\|^{2} \geqslant 2$ and if $T$ is compact then SYNTH1 stops locally (but not globally) earlier than the implicit Euler scheme.

Proof . Let $\left\{w_{n}^{1}\right\},\left\{w_{n}^{2}\right\}$ and $R_{1}, R_{2}$ denote the sequences and stability functions of SYNTH1 and the implicit Euler method, respectively. Observe that $T w_{n}^{i}-g^{\varepsilon}=R_{i}\left(-\Delta \mathrm{t} T T^{*}\right)^{n} g^{\varepsilon}, i=$ 1, 2. Hence, (5.2) holds true with $Q_{n}=q_{n}\left(T T^{*}\right)$ where

$$
q_{n}(\lambda)=\left(\frac{R_{1}(-\Delta \mathrm{t} \lambda)}{R_{2}(-\Delta \mathrm{t} \lambda)}\right)^{n}=\left(\frac{\frac{1-\theta \Delta \mathrm{t} \lambda}{1+\Delta \lambda}}{\frac{1}{1+\Delta \mathrm{t} \lambda}}\right)^{n}=(1-\theta \Delta \mathrm{t} \lambda)^{n} .
$$


Under (a) we have $\sup _{0 \leqslant \lambda \leqslant\|T\|^{2}}\left|q_{n}(\lambda)\right|=1$ and $\lim _{n \rightarrow \infty} q_{n}(\lambda)=0$ pointwise in $\left.] 0,\|T\|^{2}\right]$ implying that $\lim _{n \rightarrow \infty} q_{n}\left(T T^{*}\right) y=0$ for all $y \in Y{ }^{8}$

To validate part (b) we construct a projection $P$ of finite rank such that the spectrum of $q_{n}\left(T T^{*}\right)(I-P)$ is contained in $q_{n}\left(\left[0, \frac{2}{\theta \Delta \mathrm{t}}[)\right.\right.$. Let $\left.\left\{\left(\sigma_{j} ; v_{j}, u_{j}\right): j \in \mathbb{N}\right\} \subset\right] 0, \infty[\times X \times Y$ be the singular system of the non-degenerate compact operator $T$, that is, $T x=\sum_{j=1}^{\infty} \sigma_{j}\left\langle x, v_{j}\right\rangle_{X} u_{j}$ with $\lim _{j \rightarrow \infty} \sigma_{j}=0$ monotonically and $\left\{v_{j}\right\}$ and $\left\{u_{j}\right\}$ are orthonormal bases in $\mathrm{N}(T)^{\perp}$ and $\overline{\mathrm{R}(T)}$, respectively. Set

$$
P y:=\sum_{j \in \mathcal{J}}\left\langle y, u_{j}\right\rangle_{Y} u_{j}, \quad \mathcal{J}:=\left\{j \in \mathbb{N}: \sigma_{j}^{2} \geqslant \frac{2}{\theta \Delta \mathrm{t}}\right\},
$$

yielding $q_{n}\left(T T^{*}\right)(I-P) y=\sum_{j \notin \mathcal{J}} q_{n}\left(\sigma_{j}^{2}\right)\left\langle y, u_{j}\right\rangle_{Y} u_{j} . \quad$ By $\sup _{0 \leqslant \lambda<\frac{2}{\theta \Delta t}}\left|q_{n}(\lambda)\right|=1$ and $\lim _{n \rightarrow \infty} q_{n}(\lambda)=0$ for $\left.\lambda \in\right] 0, \frac{2}{\theta \Delta \mathrm{t}}\left[\right.$, we end with $\lim _{n \rightarrow \infty} q_{n}\left(T T^{*}\right)(I-P) y=0$ for all $y \in Y$.

Remark 5.4. We formulated theorem 5.3 for constant time steps only. Variable time steps can also be dealt with: for instance, if $\left.\left\{\Delta \mathrm{t}_{n}\right\} \subset\right] 0, \frac{1}{\theta\|T\|^{2}}\left[\right.$ is such that $\sum_{n} \Delta \mathrm{t}_{n}$ diverges to infinity then SYNTH1 stops earlier than the implicit Euler scheme. This can be seen from $q_{n}(\lambda)=\prod_{k=1}^{n}\left(1-\theta \Delta \mathbf{t}_{k} \lambda\right)$ which converges to zero pointwise on $\left.] 0,\|T\|^{2}\right]$ under the assumptions on the sequence of step sizes.

Due to remark 4.2 we will, however, focus our attention on constant time steps only.

Theorem 5.5. Let $T$ be in $\mathcal{L}(X, Y)$ with a non-closed range. We consider SYNTH2 with $0 \leqslant \theta<2(1+\sqrt{2})$ and the implicit Euler scheme where both schemes share the same constant step size $\Delta \mathrm{t}>0$.

(a) If $(\theta-1) \Delta \mathrm{t}\|T\|^{2}<2$ then SYNTH2 stops earlier than the implicit Euler scheme. (Especially, for $\theta \leqslant 1$ SYNTH2 stops earlier for all step sizes independently of the magnitude of $\|T\|$.)

(b) If $(\theta-1) \Delta \mathrm{t}\|T\|^{2} \geqslant 2$ and if $T$ is compact then SYNTH2 stops locally (but not globally) earlier than the implicit Euler scheme.

Proof. Denoting by $\left\{w_{n}^{1}\right\}$ and $\left\{w_{n}^{2}\right\}$ the sequences of SYNTH2 and the implicit Euler scheme, respectively, (5.2) holds for $Q_{n}=q_{n}\left(T T^{*}\right)$ where

$$
q_{n}(\lambda)=\left(\frac{1-\theta \Delta \mathrm{t} \lambda}{1+\Delta \mathrm{t} \lambda}\right)^{n}
$$

We can now proceed as in the proof of theorem 5.3.

Theorem 5.6. Let $T$ be in $\mathcal{L}(X, Y)$ with a non-closed range. We consider SYNTHI and SYNTH2 where both schemes share the same $\theta \in[0,1[$ and the same constant step size $\Delta \mathrm{t}>0$

Then, SYNTH2 stops earlier than SYNTH1.

Proof. Here, $Q_{n}=q_{n}\left(T T^{*}\right)$ with $q_{n}(\lambda)=(1+\Delta \mathrm{t} \lambda)^{-n}$.

If scheme S1 stops earlier than scheme S2 then S1 requires a smaller number of iterations than $\mathrm{S} 2$ to be stopped by the discrepancy principle. But a smaller stopping index tells nothing about the overall required numerical operations, that is, about the consumed CPU-time. The correct quantities to be compared are the products of the number of iterations times the

8 First, show that $\lim _{n \rightarrow \infty} q_{n}\left(T T^{*}\right) y=0$ for all $y \in \mathrm{R}\left(T T^{*}\right)$. Since $\mathrm{R}\left(T T^{*}\right)$ is dense in $Y$ and $\left\|q_{n}\left(T T^{*}\right)\right\| \leqslant 1$, the uniform boundedness principle yields pointwise convergence. 

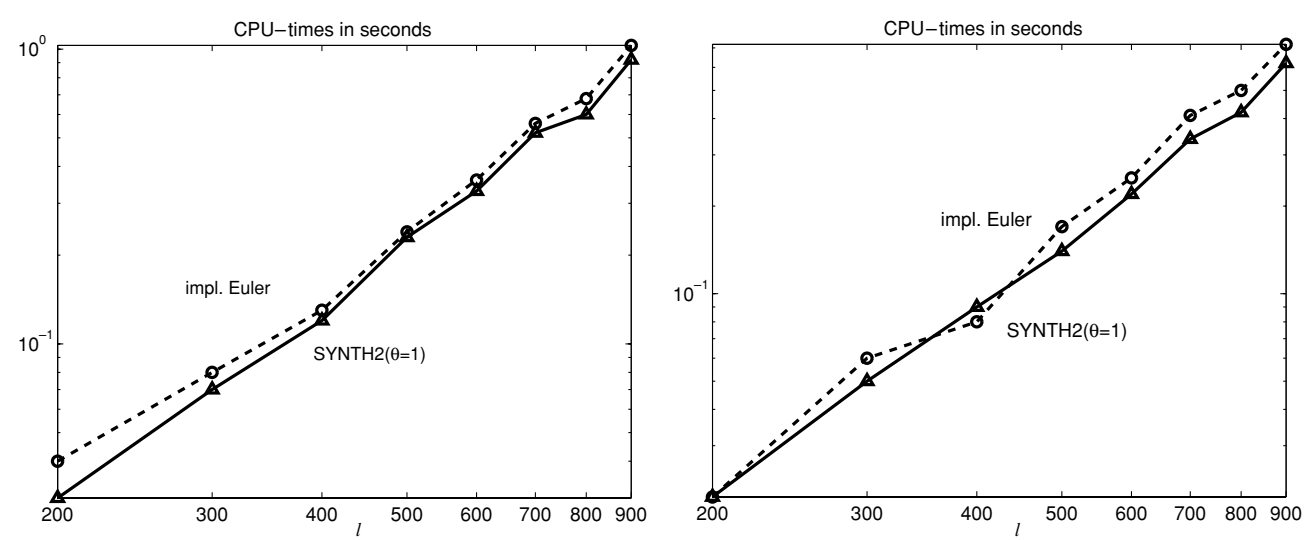

Figure 4. CPU-times for example 1 relative to SYNTH2 with $\theta=1$ (solid lines with $\triangle$ ) and the implicit Euler scheme (dashed lines with $\circ$ ). Left: noise level $\delta=h_{l}^{2}$ in (4.4) and constant time step $\Delta \mathrm{t}=l^{3 / 2}$ (same setting as underlying figure 2). Right: noise level $\delta=0.02\left\|g_{1}\right\|_{L^{2}(0,1)}$ in (4.4) and constant time step $\Delta \mathrm{t}=100$.

numerical effort per iteration. For instance, SYNTH2 with $\theta=1$ stops earlier than the implicit Euler scheme. On the other hand, one iteration step of SYNTH2 is more than twice as expensive as one implicit Euler step. Hence, SYNTH2 outperforms the Euler scheme whenever the Euler scheme requires more than twice as many iteration steps as SYNTH2.

Besides speed, accuracy also matters. Here, the error constant, which is the smallest possible constant $\widetilde{C}_{\mu}$ in the error estimate of theorem 4.1 , tells us which method we can expect to deliver the more accurate reconstruction. Unfortunately, meaningful numerical values for the error constants of SYNTH2 and the Euler scheme are difficult to obtain and therefore we leave the accuracy question unanswered analytically.

We did, however, perform some numerical computations. In the left part of figure 4 we plotted CPU-times needed by both schemes to solve (4.1) with exact solution $f_{1}^{+}$(see first paragraph of section 4.2 and compare figure 2). In this considered setting SYNTH2 is indeed slightly faster than the implicit Euler iteration. We observe the very same picture when the noise level does not decrease to zero as $l \rightarrow \infty$ : figure 4 displays on its right the CPU-times for a relative noise level of $2 \%$, that is, $\delta=0.02\left\|g_{1}\right\|_{L^{2}(0,1)}$ in (4.4). Please note: in all computations underlying figure 4 both schemes produced practically identical reconstruction errors (4.5).

\section{Final remarks}

\subsection{Exponential integrators}

Not only Runge-Kutta methods can be used for regularizing (1.1) but also the so-called exponential integrators. Exponential integrators are Runge-Kutta-like schemes which solve linear ODEs exactly, see, for instance, Hochbruck, Lubich and Selhofer [7]. The simplest exponential integrator is the exponentially fitted Euler method. Applied to the autonomous system

$$
w^{\prime}(t)=\Psi(w(t)), \quad t>0, \quad w(0)=w_{0},
$$

the exponentially fitted Euler method produces the sequence

$$
w_{n}=w_{n-1}+\Delta \mathrm{t}_{n} \varphi\left(\Delta \mathrm{t}_{n} \Psi^{\prime}\left(w_{n-1}\right)\right) \Psi\left(w_{n-1}\right)
$$


where $\varphi(z)=\left(\mathrm{e}^{z}-1\right) / z$ and $\Psi^{\prime}: W \rightarrow \mathcal{L}(W)$ is the Fréchet derivative of the mapping $\Psi: W \rightarrow W$ ( $W$ Banach space). The application of exponential integrators for solving (4.1) relies on the evaluation of expressions like $\varphi\left(\Delta \mathrm{t}_{n} A\right) v$ ( $A$ and $v$ being a matrix and a vector, respectively). Hochbruck and Lubich [6] compute $\varphi\left(\Delta \mathrm{t}_{n} A\right) v$ very efficiently by Krylov subspace approximations.

As (2.3) holds correspondingly for exponential integrators so do theorems 3.2 and 3.3 (see Hönig [8]). The numerical experiments presented in [8] reveal the exponential integrators to be competitive with the implicit Runge-Kutta schemes. However, the choice of the integration step size $\Delta \mathrm{t}_{n}$ is more subtle because the larger $\Delta \mathrm{t}_{n}$ is the more Arnoldi iterations are required to obtain a useful Krylov subspace approximation to $\varphi\left(\Delta \mathrm{t}_{n} A\right) v$.

\subsection{Nonlinear problems}

Asymptotic regularization is defined for nonlinear ill-posed problems as well (see Tautenhahn [14]). Assume that the operator $T$ in (1.1) is nonlinear and Fréchet-differentiable. Then asymptotic regularization means solving the evolution equation

$$
u^{\prime}(t)=T^{\prime}(u(t))^{*}\left(g^{\varepsilon}-T(u(t))\right), \quad u(0)=u_{0},
$$

and taking $u\left(t_{\text {stop }}\right)$ for a selected stopping time $t_{\text {stop }}$ as the stable approximate solution of the unperturbed problem $T(f)=g$.

The application of Runge-Kutta or exponential integrators to the above ODE offers a variety of new potential regularization schemes for nonlinear ill-posed problems. A good deal of work remains to be done.

\section{Appendix. The RKCs iteration scheme}

Following the ideas of van der Houwen and Sommeijer [15] we construct the iteration scheme (3.12) starting from the stability function $R(z)=\mathrm{T}_{s}\left(\omega_{0}+\omega_{1} z\right) / \mathrm{T}_{s}\left(\omega_{0}\right)$.

We rely on the three-term recursion for the first kind Chebyshev polynomials: $T_{0}(z)=1$, $\mathrm{T}_{1}(z)=z$,

$$
\mathrm{T}_{j}(z)=2 z \mathrm{~T}_{j-1}(z)-\mathrm{T}_{j-2}(s), \quad j=2,3, \ldots
$$

Let $R_{j}(z)=\mathrm{T}_{j}\left(\omega_{0}+\omega_{1} z\right) / \mathrm{T}_{j}\left(\omega_{0}\right), j=0, \ldots, s$. Then, $R_{s}(z)=R(s)$, and, by (A.1), we have the recursion: $R_{0}(z)=1, R_{1}(z)=1+\tilde{\mu}_{1} z$,

$$
R_{j}(z)=\mu_{j} R_{j-1}(z)+\tilde{\mu}_{j} z R_{j-1}(z)+v_{j} R_{j-2}(z), \quad j=2, \ldots, s,
$$

where the coefficients $\left\{\mu_{j}\right\}_{2 \leqslant j \leqslant s},\left\{\tilde{\mu}_{j}\right\}_{2 \leqslant j \leqslant s},\left\{v_{j}\right\}_{2 \leqslant j \leqslant s}$ are those from (3.13). Taking into account that $\mu_{j}+v_{j}=1, j=2, \ldots, s$, we obtain also a recursion for the polynomials $Q_{j}(z)=\left(R_{j}(z)-1\right) / z: Q_{0}(z)=0, Q_{1}(z)=\tilde{\mu}_{1}$,

$$
Q_{j}(z)=\mu_{j} Q_{j-1}(z)+\tilde{\mu}_{j} R_{j-1}(z)+v_{j} Q_{j-2}(z), \quad j=2, \ldots, s .
$$

Define $w_{n, j}:=R_{j}\left(-\Delta \mathrm{t}_{n} T^{*} T\right) w_{n-1}+\Delta \mathrm{t}_{n} Q_{j}\left(-\Delta \mathrm{t}_{n} T^{*} T\right) T^{*} y, j=0, \ldots, s$. Then, $w_{n, 0}=$ $w_{n-1}, w_{n, 1}=w_{n, 0}+\tilde{\mu}_{1} \Delta \mathrm{t}_{n} T^{*}\left(y-T w_{n, 0}\right)$, and

$$
w_{n, j}=\mu_{j} w_{n, j-1}+v_{j} w_{n, j-2}+\tilde{\mu}_{j} \Delta \mathrm{t}_{n} R_{j-1}\left(-\Delta \mathrm{t}_{n} T^{*} T\right) T^{*}\left(y-T w_{n-1}\right),
$$


$j=2, \ldots, s$. We further inspect the right most term. Since $R_{j-1}(z)=1+z Q_{j-1}(z)$ we deduce that

$$
\begin{aligned}
R_{j-1}\left(-\Delta \mathrm{t}_{n}\right. & \left.T^{*} T\right) T^{*}\left(y-T w_{n-1}\right) \\
& =R_{j-1}\left(-\Delta \mathrm{t}_{n} T^{*} T\right) T^{*} y-R_{j-1}\left(-\Delta \mathrm{t}_{n} T^{*} T\right) T^{*} T w_{n-1} \\
& =\left(I-\Delta \mathrm{t}_{n} T^{*} T Q_{j-1}\left(-\Delta \mathrm{t}_{n} T^{*} T\right)\right) T^{*} y-T^{*} T R_{j-1}\left(-\Delta \mathrm{t}_{n} T^{*} T\right) w_{n-1} \\
& =T^{*} y-T^{*} T\left(R_{j-1}\left(-\Delta \mathrm{t}_{n} T^{*} T\right) w_{n-1}+\Delta \mathrm{t}_{n} Q_{j-1}\left(-\Delta \mathrm{t}_{n} T^{*} T\right) T^{*} y\right) \\
& =T^{*}\left(y-T w_{n, j-1}\right) .
\end{aligned}
$$

Hence,

$$
w_{n, j}=\mu_{j} w_{n, j-1}+v_{j} w_{n, j-2}+\tilde{\mu}_{j} \Delta \mathrm{t}_{n} T^{*}\left(y-T w_{n, j-1}\right), \quad j=2, \ldots, s,
$$

and the proof of (3.12) is completed.

\section{References}

[1] Deuflhard P and Bornemann F 2002 Scientific Computing with Ordinary Differential Equations (Texts in Applied Mathematics vol 42) (Berlin: Springer)

[2] Engl H W, Hanke M and Neubauer A 1996 Regularization of Inverse Problems (Mathematics and Its Applications vol 375) (Dordrecht: Kluwer)

[3] Hairer E and Wanner G 1996 Solving Ordinary Differential Equations II (Springer Series in Computational Mathematics vol 14) (Berlin: Springer)

[4] Hanke M and Groetsch C W 1998 Nonstationary iterated Tikhonov regularization J. Opt. Theory Appl. 98 37-53

[5] Hestenes M R and Stiefel E 1952 Methods of conjugate gradients for solving linear systems J. Res. NBS 49 409-36

[6] Hochbruck M and Lubich Chr 1997 On Krylov subspace approximations to the matrix exponential operator SIAM J. Numer. Anal. 34 1911-25

[7] Hochbruck M, Lubich Chr and Selhofer H 1998 Exponential integrators for large systems of differential equations SIAM J. Sci. Comput. 19 1552-74

[8] Hönig M 2004 Asymptotische Regularisierung schlecht gestellter Probleme mittels steifer Integratoren (Regularization of ill-posed problems by stiff integrators) Diploma Thesis Fakultät für Mathematik, Universität Karlsruhe, Germany

[9] Hundsdorfer W and Verwer J G 2003 Numerical Solution of Time-Dependent Advection-Diffusion-Reaction Equations (Springer Series in Computational Mathematics vol 33) (Berlin: Springer)

[10] Plato R 1990 Über die Diskretisierung und Regularisierung schlecht gestellter Probleme (On the discretization and regularization of ill-posed problems) PhD Thesis Fachbereich Mathematik der Technischen Universität Berlin Online at URL: www.math.tu-berlin.de/ plato/promo.ps

[11] Rieder A 2003 Keine Probleme mit Inversen Problemen (No Problems with Inverse Problems) (Wiesbaden: Vieweg)

[12] Schock E 1987 Comparison principles for iterative methods Inverse and Ill-Posed Problems (Notes and Reports in Mathematics in Science and Engineering vol 4) ed H W Engl and C W Groetsch (Boston, MA: Academic) pp 185-93

[13] Schock E 1987 Implicit iterative methods for the approximate solution of ill-posed problems Bollettino UMI 7 Series 1-B 1171-84

[14] Tautenhahn U 1994 On the asymptotical regularization of nonlinear ill-posed problems Inverse Problems 10 $1405-18$

[15] van der Houwen P J and Sommeijer B P 1980 On the internal stability of explicit, $m$-stage Runge-Kutta methods for large $m$-values Z. Angew. Math. Mech. (ZAMM) 60 479-85 